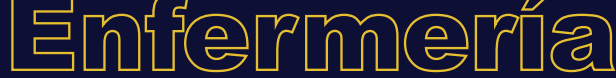

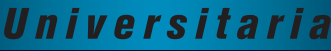

\section{Enfermería Universitaria: 15 años de contribuir a la difusión de la investigación de enfermería}

\section{Enfermería Universitaria: 15 years of contributing to the dissemination of nursing research}

\section{Enfermería Universitaria: 15 anos de contribuir para a difusão da pesquisa de enfermagem.}

Con el primer número de este año iniciamos el volumen 15 de Enfermería Universitaria, son 15 años sin interrupción de que la revista colabora en la difusión de la actividad de investigación del profesional de enfermería de este país. También, en forma paulatina, se han incorporado artículos provenientes de otras regiones de América Latina, en particular en los últimos cuatro años.

La permanencia de la publicación de Enfermería Universitaria en este lapso es muestra del apoyo de los directivos de la Escuela Nacional de Enfermería y Obstetricia (ENEO-UNAM), para que los docentes publiquen los resultados de la investigación que realizan y contribuyan a incrementar el conocimiento de la disciplina.

La revista en su evolución y con la constante mejora en la calidad del proceso editorial ha logrado consolidarse y adquirir reconocimiento, de tal forma que en la actualidad está incluida en ocho bases de datos, entre estas, las tres principales de América Latina: La Biblioteca Virtual en Salud Regional, Scielo y Redalyc, en las dos últimas es la única revista de enfermería de México. En 2016 obtiene otro reconocimiento importante, al ser incluida en el Sistema de Clasificación de Revistas Mexicanas de Ciencia y Tecnología del Consejo Nacional de Ciencia y Tecnología de nuestro país. Estos datos están íntimamente relacionados con la visibilidad y difusión alcanzada, por ejemplo, durante 2017 el número de visitas al sitio de la revista, sólo en la base de datos Scielo, fue de 481 mil, lo que equivale a 1317 visitas por día.

Celebrar estos 15 años de permanencia, es motivo para realizar un recuento de lo publicado e identificar el tipo de artículos; los diseños utilizados en los artículos de investigación; cuáles son las áreas de interés, qué poblaciones de estudio son prioritarias para enfermería; y, finalmente cuál es la procedencia de los autores.

Con este propósito se realizó un análisis para el cual, del total de escritos publicados se excluyeron las editoriales y escritos informativos, reseñas o de opinión, incluidos básicamente en el primer periodo delimitado; a partir de 2010 se establecieron las secciones consideradas en la revista hasta la actualidad: a) artículos de investigación -originales-, b) de revisión y c) de innovación para la práctica; conforme a estas se clasificaron todos los artículos; que independientemente de la sección deben 
fundamentarse en metodología específica al tipo de artículo. Los periodos establecidos corresponden a tres diferentes gestiones que ha tenido la revista, el primero de 2004 a 2007, el segundo de 2008 a 2011 y el último de 2012 a la fecha. La clasificación del tipo de diseño en estudios cuantitativos respondió al grado de complejidad en el análisis estadístico y en los cualitativos se incluyeron los principales diseños considerados. Las áreas se delimitaron a partir de las contenidas para la investigación en enfermería en los Descriptores en Ciencias de la Salud. Respecto a las poblaciones de estudio en primer lugar se consideró la clasificación según etapa del ciclo vital, adicionalmente se concretaron otros grupos en función de la frecuencia en que fueron considerados en las investigaciones.

El total de artículos publicados entre 2004-2017 fue de 318, es hasta los dos últimos años en que se incrementa el total de artículos incluidos por número. En la revista se consideran básicamente tres secciones de acuerdo al tipo de artículo, pero en la tabla 1 se decidió mostrar la información más desagregada. La mitad del total de artículos corresponde a investigación cuantitativa, 11\% a cualitativa, un porcentaje similar de artículos de revisión y la cuarta parte restante la constituyen los artículos comprendidos en la sección de innovación para la práctica: procesos de atención de enfermería/estudios de caso, ensayos o experiencias de innovación para la práctica.

\begin{tabular}{|c|c|c|c|c|c|c|c|c|}
\hline \multirow[t]{2}{*}{ Tipo de Artículo } & \multicolumn{2}{|c|}{ 2004-2007 } & \multicolumn{2}{|c|}{ 2008-2011 } & \multicolumn{2}{|c|}{ 2012-2017 } & \multicolumn{2}{|c|}{ Total } \\
\hline & F & $\%$ & $\mathrm{~F}$ & $\%$ & F & $\%$ & $\mathrm{~F}$ & $\%$ \\
\hline Inv. Cuantitativa & 27 & 41.5 & 56 & 59.0 & 74 & 46.8 & 157 & 49.4 \\
\hline Inv. Cualitativa & 5 & 7.7 & 6 & 6.3 & 23 & 14.6 & 34 & 10.7 \\
\hline Inv. Mixta & 1 & 1.5 & 0 & 0 & 3 & 1.9 & 4 & 1.2 \\
\hline Revisión & 8 & 12.3 & 10 & 10.5 & 26 & 16.4 & 44 & 13.8 \\
\hline PAE/Est de caso & 3 & 4.6 & 4 & 4.2 & 19 & 12.0 & 26 & 8.2 \\
\hline Ensayo & 12 & 18.6 & 12 & 12.6 & 8 & 5.1 & 32 & 10.1 \\
\hline Exp de innovación & 9 & 13.8 & 7 & 7.4 & 5 & 3.2 & 21 & 6.6 \\
\hline Total & 65 & 100 & 95 & 100 & 158 & 100 & 318 & 100 \\
\hline
\end{tabular}

Conforme a lo deseado para una revista científica, desde el segundo periodo se han incluido en mayor medida artículos de investigación; en el último periodo en comparación con los anteriores se duplicó el porcentaje de artículos de investigación cualitativa, hubo aumento en los de revisión y los Procesos de Atención de Enfermería o Estudios de caso; han disminuido aquellos referidos a ensayos y a experiencias de innovación para la práctica.

Respecto al tipo de diseño en la Tabla 2, en las investigaciones cuantitativas, se observa el predominio de diseños descriptivos, dos terceras partes corresponden a este tipo; poco más de $20 \%$ fueron estudios de asociación o correlación y con porcentajes iguales o menores a 5\% otro tipo de diseños. En los estudios cualitativos el mayor porcentaje fueron de tipo fenomenológico. En este caso, otro tipo de estudios aglutinó la tercera parte de ellos. Los artículos de tipo cualitativo se incrementaron a partir de 2012, pero en mayor medida en los últimos dos años.

Respecto a las áreas principales abordadas en mayor medida, hubo artículos relacionados con Cuidados de enfermería (39\%) y al interior de estos 16\% corresponde a atención primaria (salud comunitaria, salud en el hogar, de la familia, de salud pública), 23\% a la atención clínica con una variedad en 19 áreas de enfermería, entre estas destacan por su frecuencia enfermería geriátrica, obstétrica y de cuidados críticos. El otro ámbito que sigue en importancia son artículos relativos a la Educación de enfermería (25\%). 
Tabla 2. Tipo de diseño

\begin{tabular}{lcc}
\hline & Frecuencia & Porcentaje \\
\cline { 2 - 3 } Cuantitativo: & 106 & 67.5 \\
\hline Descriptivo & 34 & 21.7 \\
Estudio de asociación & & \\
-correlación & & 5.1 \\
Estudio de intervención & 7 & 4.4 \\
Validación de & 2 & 1.3 \\
Instrumentos & 157 & 82.2 \\
Epidemiológico & & \\
Subtotal & 15 & 44.1 \\
Cualitativo: & 2 & 5.9 \\
\hline Fenomenológico & 3 & 8.8 \\
Etnografía & 3 & 8.8 \\
Teoría fundamentada & 11 & 32.4 \\
Investigación-acción & 34 & 17.8 \\
Otros & 191 & 100.0 \\
Subtotal & & \\
Total & & \\
\hline & & \\
\hline
\end{tabular}

Tabla 3. Área Principal en los

artículos publicados

\begin{tabular}{|c|c|c|c|}
\hline Principal & Secundaria & Frecuencia & Porcentaje \\
\hline \multirow{2}{*}{$\begin{array}{l}\text { Cuidados de } \\
\text { Enfermería }\end{array}$} & $\begin{array}{l}\text { Atención } \\
\text { Primaria* }\end{array}$ & 50 & 15.7 \\
\hline & $\begin{array}{l}\text { Atención } \\
\text { Clínica** }\end{array}$ & 73 & 23.0 \\
\hline \multirow[t]{2}{*}{$\begin{array}{l}\text { Gestión de } \\
\text { Enfermería }\end{array}$} & $\begin{array}{c}\text { Evaluación } \\
\text { de la } \\
\text { Calidad } \\
\text { de los } \\
\text { servicios } \\
\text { Salud }\end{array}$ & 43 & 13.5 \\
\hline & $\begin{array}{l}\text { Salud } \\
\text { laboral }\end{array}$ & 12 & 3.8 \\
\hline $\begin{array}{l}\text { Educación de } \\
\text { Enfermería }\end{array}$ & & 79 & 24.8 \\
\hline $\begin{array}{l}\text { Investigación } \\
\text { Metodológica }\end{array}$ & & 39 & 12.3 \\
\hline \multirow[t]{2}{*}{ Otras } & & 22 & 6.9 \\
\hline & Total & 318 & 100.0 \\
\hline
\end{tabular}

Lo más destacable en la tabla 4, es que en poco menos de la mitad de los artículos, la población de estudio la constituyen profesionales, estudiantes, pasantes o docentes de enfermería, en cambio en los otros grupos, objeto de cuidado de enfermería, los porcentajes son menores al $10 \%$, sobresalen adultos, adultos mayores y mujeres, aunque hay un 15\% de artículos en que se considera a población de diversas edades.

\begin{tabular}{lcc} 
Tabla 4. Población estudiada & & \\
\hline Población & Frecuencia & Porcentaje \\
\hline Pre/ escolar & 6 & 3.1 \\
\hline Adolescentes & 4 & 2.1 \\
\hline Adulto & 12 & 6.8 \\
\hline Adulto Mayor & 15 & 7.9 \\
\hline Mujeres (Adultas/adolescentes) & 15 & 7.9 \\
\hline Población obstétrica & 10 & 5.2 \\
\hline Cuidador familiar & 8 & 4.2 \\
\hline Familia & 2 & 1.0 \\
\hline Prof. de Enfermería & 46 & 23.6 \\
\hline Estudiantes/Pasantes de Enfermería & 33 & 17.3 \\
\hline Docentes de Enferm & 5 & 2.6 \\
\hline Otros & 5 & 2.6 \\
\hline Varias edades o participantes & 30 & 15.7 \\
\hline Total & 191 & 100 \\
\hline
\end{tabular}


En la Gráfica 1 se presenta la diversidad en la procedencia de los autores en el último periodo considerado, aunque se incluye un año del periodo previo que es representativo de los dos primeros periodos. Se observa que a partir de 2013 aumenta la participación de autores de otros estados de la república mexicana, así como de otros países, hasta alcanzar en el último año más del 40\% de autores de diferentes naciones. Es preciso detenerse en otro aspecto importante como lo es la proporción de estudios colaborativos, que en este caso se ubican en ENEO/Otros, aunque presentes, son mínimos.

Gráfica 1. Enfermería Universitaria: Procedencia de los autores en artículos publicados 2011-2017

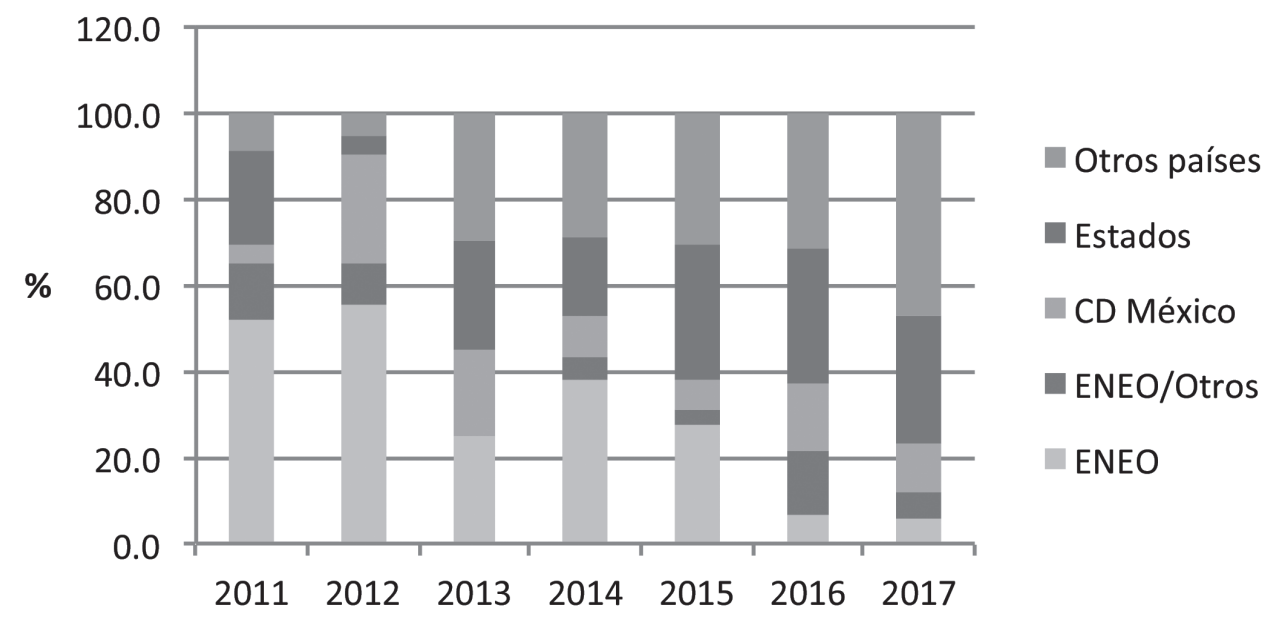

Conforme a este panorama obtenido es posible apreciar que en el total de artículos publicados, de acuerdo a lo deseable, se ha mantenido el porcentaje de los derivados de proyectos de investigación. Al considerar el tipo de diseño en los estudios cuantitativos, se observa el predominio de estudios descriptivos y un avance incipiente, pues otra cuarta parte proviene de investigaciones correspondientes a estudios de asociación o correlación, es mínima la proporción de estudios de intervención. Las áreas principales reflejan una adecuada diversidad, pues el mayor porcentaje corresponde a aspectos vinculados con el cuidado de enfermería ya sea en atención primaria o en atención clínica; de igual modo, es favorable el interés por temáticas actuales como es la evaluación de la calidad de los servicios de salud, y la incursión en la evaluación de aspectos metodológicos.

Los artículos sobre educación de enfermería, constituyen otro grupo significativo de abordaje, por una parte son propios del nicho de la revista, inserta en una comunidad universitaria y por otro, pueden ser vinculados con un alto porcentaje identificado aquí, donde la población de estudio es personal de enfermería, estudiantes, pasantes y docentes de enfermería; igual ocurre con los artículos que evalúan la calidad de la atención cuya población de estudio puede ser el propio personal de enfermería. Este fenómeno se ha documentado ampliamente, Jacqueline Fawcett en 2014 al evaluar las tendencias de investigación señala: la mayoría de los temas de investigación desde los años 20 hasta finales de los 60 [del siglo XX] se centraron en la educación en enfermería y en las enfermeras... La investigación sobre la educación en enfermería continúa hoy en día, pero en menor medida que en el pasado ${ }^{1}$. Igual ocurre con los estudios de gestión realizados con personal de enfermería.

De la misma manera, el uso predominante del diseño descriptivo ha sido identificado desde 1990, tanto en la investigación realizada en México como en la de otras regiones de Iberoamérica ${ }^{2-4}$. Los datos mostrados aquí confirman su persistencia.

Los trabajos provenientes de investigación cualitativa aunque se han incrementado, no alcanzan siquiera el porcentaje observado entre 2002-2007, en la Revista Avances de Enfermería (21\%) y en 
la Revista Latinoamericana de Enfermagem $(49 \%)^{5}$. Es decir este tipo de investigación aún requiere mayor desarrollo en nuestro medio.

\section{Reflexiones finales}

Los datos presentados reflejan avances importantes en Enfermería Universitaria, que se fundamentan en su calidad editorial, esto ha posibilitado que la revista se constituya en un referente para enfermería a nivel nacional y en años recientes también internacional, por consiguiente cumple con la Misión establecida de Contribuir a la difusión del quehacer científico de enfermería en México y en países de habla hispana; asimismo mantiene su Visión de Posicionarse como una de las principales revistas de enfermería en Iberoamérica.

Igualmente, este panorama proporciona valiosa información para que los encargados de coordinar la investigación en diferentes ámbitos fundamenten cambios necesarios en los protocolos que se realizan; se requiere continuar fortaleciendo las herramientas metodológicas de forma que se avance en diseños de mayor complejidad y en perspectivas diferentes, como sería la investigación cualitativa; aunque en la revista por formar parte de una comunidad universitaria permanezca el énfasis de las investigaciones en educación de enfermería, es conveniente favorecer que los estudios provenientes del área clínico-asistencial se orienten en mayor medida a la población usuaria de los servicios más que al personal mismo. Finalmente, es preciso promover la elaboración de estudios colaborativos y multidisciplinarios que al mismo tiempo de la investigación coadyuven a compartir aprendizajes y avances de otras áreas y regiones.

\section{Referencias}

1. Fawcett J. Tendencias de investigación en enfermería. Aquichan 2014; 14(3):289-92.

2. García-Rodríguez M, Gómez-Ávila MG, Aguilar-Pérez I, et al. Tendencias y características de la investigación en enfermería publicada en México. Enferm. Univ. 2011; 8(1): 7-16.

3. Manfredi M. La investigación en enfermería en América Latina. Educ Med Salud 1991; 25(2): 154-66.

4. Cometo MC, Piovan M, Gómez P. Aportes de los coloquios panamericanos a la investigación en enfermería - período 2000-2006. Texto Contexto Enferm. 2008; 17 (4):720-6.

5. Díaz Heredia LP, Bueno Robles LS, Mejía Rojas ME, et al. Investigación de enfermería: una caracterización en dos publicaciones latinoamericanas. Av.enferm. 2011; XXIX (2): 285-93.

M. García-Cardona*

I.D. Cruzaley-Castro

Escuela Nacional de Enfermería y Obstetricia

*rev. enfermeriauniversitaria@gmail.com 